\title{
MODELAGEM E SIMULAÇÃO DE REATORES RISER PARA CRAQUEAMENTO CATALÍTICO DE PETRÓLEO UTILIZANDO UM SOFTWARE COMERCIAL
}

\author{
F. J. QUEIROZ ${ }^{1}$, B. C. C. SANTOS ${ }^{2}$, P. L. BARROS ${ }^{1}$ e J. J. N. ALVES ${ }^{1}$ \\ ${ }^{1}$ Universidade Federal de Campina Grande, Unidade Acadêmica de Engenharia Química \\ ${ }^{2}$ Universidade Federal de Campina Grande, Unidade Acadêmica de Engenharia de Petróleo \\ E-mail para contato: josimar.queiroz@eq.ufcg.edu.br
}

\begin{abstract}
RESUMO - O craqueamento catalítico de petróleo é um dos processos mais importantes da indústria petrolífera, no qual frações pesadas de óleo são convertidas em outros produtos de maior valor agregado. Com a modelagem e simulação de reatores do tipo riser utilizando o software ANSYS CFX foi possível analisar a produção de gasolina, gases leves e coque a partir do craqueamento catalítico do gasóleo de vácuo baseado no modelo cinético de 4-lumps. A simulação numérica foi validada através da comparação com os dados de uma planta industrial disponíveis na literatura, o que mostra que o modelo adotado foi adequado. A influência do parâmetro operacional razão catalisador e fração de óleo (gasóleo de vácuo) na alimentação foi estudada. Os resultados obtidos nos mostram a versatilidade de operação desse tipo de unidade, que pode alternar a sua produção conforme as demandas do mercado.
\end{abstract}

\section{INTRODUÇÃO}

O craqueamento de petróleo é um processo antigo, mas que ainda continua sendo objeto de estudo por ser uma das etapas mais importantes da indústria de petróleo. A fluidodinâmica computacional é uma ferramenta que pode trazer resultados satisfatórios, uma vez que com ela é possível estudar detalhadamente o processo e, consequentemente, apontar possíveis melhorias.

\subsection{Craqueamento catalítico do petróleo}

$\mathrm{O}$ craqueamento catalítico surgiu no início do século XX juntamente com o desenvolvimento da indústria automobilística nos Estados Unidos, e passou a ser cada vez mais utilizado durante a Segunda Guerra Mundial com o conflito em andamento visando atender a demanda crescente por gasolina. É um processo de refino que tem como objetivo converter frações pesadas de hidrocarbonetos provenientes da destilação de petróleo (gasóleo de vácuo) em produtos de maior valor agregado e apelo comercial (gasolina, GLP, gasóleo e outros), que serão utilizados como combustíveis ou matéria-prima de produtos químicos e/ou plásticos. 


\subsection{Reator riser}

O reator riser vem sendo utilizado como o principal reator comercial para o processo de craqueamento catalítico de petróleo ao longo das últimas décadas (Wu et al., 2009). É um reator tubular, de leito fluidizado, o tempo de residência é baixo, e que é submetido a elevadas temperatura e turbulência. As unidades industriais possuem pequenos diâmetros $(0,8 \mathrm{~m}-1,2$ m) e o comprimento geralmente varia de 30 a 40 metros (Gupta et al., 2010), a razão comprimento e diâmetro para esse tipo de reator é elevada.

\subsection{Modelos cinéticos}

Muitas reações ocorrem durante o processo de FCC, e os seus produtos consistem da mistura de muitos componentes. A cinética é de difícil estudo pela mútua interação na alimentação e quantidades envolvidas (Ruszowski et al., 2006). Diante disso, os pesquisadores desenvolveram modelos cinéticos agrupando um grande número de componentes em grupos menores de pseudocomponentes, chamados de lumps, que são frações distintas ou agrupamentos de classes com características similares entre si, como massa molecular e ponto de ebulição (Cianella e Alves, 2012). O modelo de 4-lumps, proposto por Lee et al. (1989), foi utilizado neste trabalho, e está representado na Figura 1.

Figura 1 - Modelo cinético de 4-lumps

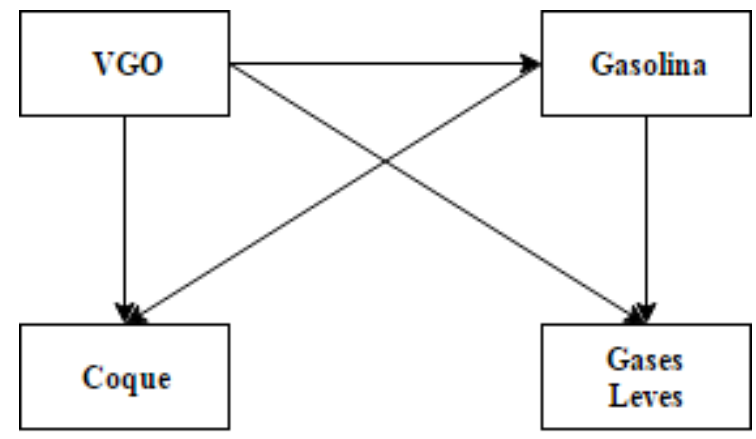

Fonte: Adaptada de Lee et al. (1989).

Os componentes são divididos em quatro grupos: VGO, gasolina, gases leves e coque. As reações de craqueamento de VGO são de segunda ordem, já as de craqueamento da gasolina são de primeira ordem.

\section{MODELAGEM MATEMÁTICA}

Os balanços de massa, momento e energia regem o processo. As taxas de consumo de um reagente j (VGO ou gasolina para o modelo cinético de 4-lumps) por unidade de volume de catalisador são calculadas de acordo Equação 1, proposta por Pachovsky e Wojciechowski (1971), e que é válida conforme mostra o trabalho de Gupta e Rao (2001).

$$
-r_{j}=\Phi_{i} k_{j}\left(\frac{C_{j}}{C_{j_{o}}}\right)^{n} C_{j}
$$


Para o craqueamento de VGO $n$ é igual a 1 , j para o craqueamento de gasolina $n$ é igual a 0 (Gupta e Rao, 2001). O termo de atividade do catalisador, baseado na concentração de coque no catalisador, é calculado a partir da Equação 2.

$$
\Phi_{i}=\frac{B+1}{B+e^{A \cdot w_{C}}}
$$

As constantes A e B da Equação 2 são, respectivamente, 4,29 e 10,4, reportadas por Pitault et al. (1995). A fração mássica de coque, produto indesejado das reações de conversão de VGO e gasolina, varia ao longo do reator. Com a Lei de Arrhenius podemos calcular a variação da constante de velocidade apresentada na equação (1):

$$
k_{j}=k_{j}^{o} e^{\left(-\frac{E_{a j}}{R}\right)\left(\frac{1}{T_{o}}-\frac{1}{T}\right)}
$$

\section{MATERIAIS E MÉTODOS}

O software ANSYS CFX, foi utilizado como ferramenta de cálculo. Considerou-se escoamento isotérmico para ambas as fases por fins de simplificação. O modelo de escoamento multifásico adotado foi o Euleriano-Euleriano, também chamado de modelo de dois fluídos. O modelo de turbulência k- $\varepsilon$ foi utilizado. Utilizou-se os dados reais de uma planta industrial reportados por Ali et al. (1997). Na Tabela 1 estão dispostas as dimensões do riser e condições operacionais, e características do catalisador.

Tabela 1 - Dimensões do riser e condições operacionais, e características do catalisador.

\begin{tabular}{|c|c|}
\hline Variável & Valor \\
\hline \hline Altura do riser $(\mathrm{m})$ & 33 \\
\hline Comprimento do riser $(\mathrm{m})$ & 0,8 \\
\hline Fluxo mássico de VGO $(\mathrm{kg} / \mathrm{s})$ & 20 \\
\hline Temperatura de VGO na alimentação $(\mathrm{K})$ & 494 \\
\hline Fluxo mássico de catalisador $(\mathrm{kg} / \mathrm{s})$ & 144 \\
\hline Temperatura do catalisador na alimentação $(\mathrm{K})$ & 960 \\
\hline Pressão $($ atm $)$ & 2,9 \\
\hline Diâmetro do catalisador $(\mu \mathrm{m})$ & 75 \\
\hline Densidade do catalisador $\left(\mathrm{kg} / \mathrm{m}^{3}\right)$ & 1200 \\
\hline
\end{tabular}

Os valores de entalpia, energia de ativação e fator pré-exponencial das reações de craqueamento catalítico para o modelo de 4-lumps utilizados estão disponíveis na literatura, respectivamente em Lee et al. (1989), Han e Chung (2000) e Pitault et al. (1995).

Analisou-se a influência da razão catalisador e VGO no fluxo mássico de alimentação. A planta opera com a razão catalisador e VGO é igual a 7,2, alterou-se essa razão para os valores de 4, 5, 6, 8, 9 e 10, mantendo o fluxo mássico alimentação constante. A concentração inicial de VGO, variável presente na Equação 1, será diferente para cada uma das razões, influenciando, consequentemente, a formação dos diferentes produtos.

A geometria e malha utilizadas nos experimentos numéricos são mostradas na Figura 2a, e a seção transversal para melhor visualização é mostrada na Figura 2-b. A malha numérica tem aproximadamente 80.000 elementos. 
Figura 2 - a) Visualização geometria e malha. b) Seção da área transversal



\section{RESULTADOS E DISCUSSÕES}

Os resultados obtidos para as composições mássicas de VGO, gasolina, gases leves e coque ao longo do reator são mostradas na Figura 3.

Figura 3 - Composição mássica dos componentes ao longo do reator

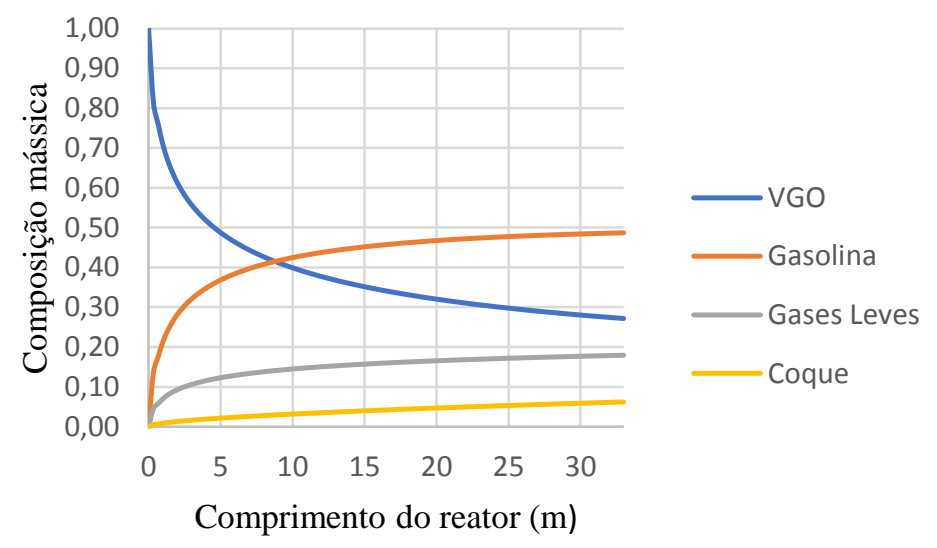

A Tabela 2 mostra os valores obtidos para a gasolina, coque e temperatura na saída do reator e erro observado quando comparados com os dados da planta.

Tabela 2 - Dados da simulação, planta e variação.

\begin{tabular}{|c|c|c|c|}
\hline Variável & Simulação & Planta & Variação \\
\hline \hline Conversão de gasolina (\%) & 48,73 & 43,9 & $9,91 \%$ \\
\hline Conversão de coque (\%) & 6,26 & 5,8 & $7,35 \%$ \\
\hline Temperatura na saída do reator (K) & 801 & 795 & $0,75 \%$ \\
\hline
\end{tabular}

A variação observada para a temperatura de saída comparando-se os dados experimentais e simulação foi mínima, menor que 1\%, resultado extremamente satisfatório. Houve uma proximidade para os valores encontrados para a conversão de gasolina e coque, o valor encontrado para a conversão de coque foi mais preciso. 
Na Figura 4 temos as composições mássicas na saída do reator para VGO não-reagido, gasolina, gases leves e coque para a razão catalisador e VGO igual 4, 5, 6, 7,2 (caso padrão), 8,9 e 10 .

Figura 4 - Composição mássica dos componentes na saída do reator para o reator padrão e diferentes razões catalisador e VGO

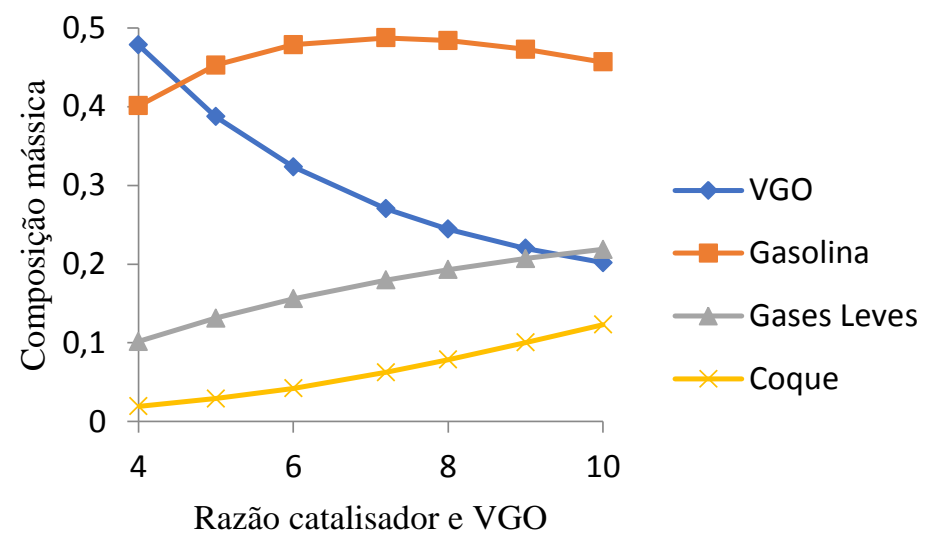

Na Tabela 3 temos os valores acima apresentados, para melhor efeito de comparação.

Tabela 3 - Composição percentual dos componentes na saída do reator para o reator padrão e diferentes razões catalisador e VGO.

\begin{tabular}{|c|c|c|c|c|c|c|c|}
\hline & $\theta=4$ & $\theta=5$ & $\theta=6$ & $\theta=7,2$ & $\theta=8$ & $\theta=9$ & $\theta=10$ \\
\hline \hline Gasolina (\%) & 40,09 & 45,24 & 47,85 & 48,73 & 48,37 & 47,26 & 45,66 \\
\hline Gases leves (\%) & 10,16 & 13,14 & 15,60 & 17,98 & 19,31 & 20,70 & 21,86 \\
\hline Coque (\%) & 1,95 & 2,91 & 4,22 & 6,26 & 7,88 & 10,03 & 12,31 \\
\hline VGO não-reagido (\%) & 47,81 & 38,71 & 32,33 & 27,03 & 24,44 & 22,01 & 20,16 \\
\hline
\end{tabular}

A quantidade produzida de gasolina para o caso padrão é superior que a quantidade obtida mesmo em simulações em que se há proporcionalmente mais catalisador, uma vez que esse aumento pode influenciar mais positivamente a produção de gases leves assim como a de coque. A razão catalisador e VGO quando igual a 8, 9 e 10 são condições operacionais mais favoráveis se levarmos em conta apenas o total de produtos utilizáveis produzido. Todavia, para os dois últimos casos ocorre um aumento mais considerável na quantidade de coque (que pode levar a desativação do catalisador, afetando consideravelmente o desempenho do processo) e uma maior diminuição na quantidade produzida de gasolina, além de se utilizar mais catalisador.

\section{CONCLUSÕES}

Os resultados provenientes da simulação estão acordo com os dados da planta disponíveis na literatura, o que indica que a modelagem de 4-lumps utilizando o software ANSYS CFX para o craqueamento catalítico de petróleo está adequada.

Foi possível perceber a grande influência do parâmetro operacional razão catalisador e VGO na alimentação do reator, influenciando a formação de produtos de interesse (gasolina e gases leves), bem como influenciando no aparecimento do produto indesejado coque. A razão 
catalisador e VGO igual a 8, e não 7,2 como utilizou-se na planta, pode apresentar uma maior possibilidade de lucros e capacidade de atender possíveis demandas da indústria petroquímica por produtos como propileno. Uma análise econômica e de mercado detalhada apontará sob que condições a operação da planta é mais vantajosa, essa versatilidade operacional é justamente um fator bastante favorável para as unidades de craqueamento catalítico de petróleo.

\section{NOMENCLATURA}

FCC Craqueamento catalítico de petróleo

$\Phi_{i} \quad$ Fator de atividade do catalisador

$w_{C} \quad$ Fração mássica de coque

$C_{j} \quad$ Concentração do componente $\mathrm{j}\left(\frac{\mathrm{mol}}{\mathrm{L}}\right)$

$C_{j_{o}} \quad$ Concentração inicial do componente puro j $\left(\frac{m o l}{L}\right)$

$k_{j} \quad$ Constante de velocidade $\left(s^{-1}\right)$

$\mathrm{R}$ Constante universal dos gases $\left(\frac{J}{\text { mol.K }}\right)$
$E_{a_{j}} \quad$ Energia de ativação de uma reação j $\left(\frac{J}{m o l . K}\right)$

$k_{j}^{o} \quad$ Fator pré-exponencial $\left(s^{-1}\right)$

VGO Gasóleo de vácuo

$\theta \quad$ Razão catalisador e gasóleo de vácuo

$r_{j} \quad$ Taxa de consumo de um reagente $\mathrm{j}$ por unidade de volume de catalisador $\left(\frac{\mathrm{kg}}{\mathrm{s}}\right)$

$T \quad$ Temperatura $(K)$

$T_{o} \quad$ Temperatura de referência

\section{REFERÊNCIAS}

ALI, H.; ROHANI, S. ; CORRIOU, J. P. Modelling and control of a riser type fluid catalytic cracking (FCC) unit. Trans IChemE, v. 75, p. 401-412, 1997.

CIANELLA, S.; ALVES, J. J. N. Modelagem e simulação do processo de craqueamento catalítico do gasóleo aplicando cálculo variacional para determinação do perfil ótimo de temperatura. I Encontro Nacional de Educação, Ciência e Tecnologia. Campina Grande. 2012.

GUPTA, R. K.; KUMAR, V.; SRIVASTAVA, V. K. Modeling of fluid catalytic cracking riser reactor: a review. International Journal of Chemical Reactor Engineering, v. 8, 2010.

GUPTA, A.; RAO, D. S Model for the performance of a fluid catalytic cracking (FCC) riser. Chemical Engineering Science, v. 56, p. 4489-4503, 2001.

HAN, I. S.; CHUNG, C. B. Dynamic modeling and simulation of a fluidized catalytic cracking process Part I: Process modeling. Chemical Engineering Science, v. 56, p. 1951-1971, 2011.

LEE, L.; CHEN, Y.; HUANG, T. Four-lump kinetic model for fluid catalytic cracking process. The Canadian Journal of Chemical Engineering, v. 67, p. 615-619, 1989.

PITAULT, I., FORISSIER, M., \& BERNARD, J. R. Determination de constantes cinetiques $\mathrm{du}$ craquage catalytique par la modelisation du test de microactivite (MAT). The Canadian Journal of Chemical Engineering, v. 73, p. 498-503, 1995.

RUSZKOWSKI, M. F.; GOMZI, Z.; TOMIC, T. 4-Lump kinetic model for hydrotreated gas oil catalytic cracking. Chemical and Biochemical Engineering Quarterly, v. 20, p. 6168, 2006.

WU, C.; CHENG, Y.; JIN, Y. Downer-to-riser coupling technique for petroleum refining. Chemical Engineering Technology, v. 3, p. 482-491, 2009. 